\title{
Predicting the gas-liquid critical point from the second virial coefficient
}

\author{
G. A. Vliegenthart and H. N. W. Lekkerkerker ${ }^{a}$ ) \\ Van 't Hoff Laboratory for Physical and Colloid Chemistry, Debye Institute, Utrecht University, \\ Padualaan 8, 3584 CH Utrecht, The Netherlands
}

(Received 8 September 1999; accepted 29 December 1999)

\begin{abstract}
We show that whereas the critical point is very sensitive to the range of interaction, the second virial coefficient has a fairly constant value at the critical temperature. This enables us to predict the critical temperature with fair accuracy. We discuss the connection between the second virial coefficient as a predictor for the critical temperature and the second virial coefficient as predictor in crystal growth as was proposed by George and Wilson [A. George and W. W. Wilson, Acta Crystallogr., Sec. D: Biol. Crystallogr. 50, 361 (1994)]. (C) 2000 American Institute of Physics. [S0021-9606(00)51212-4]
\end{abstract}

\section{INTRODUCTION}

In recent years, it has become clear that below a critical range of the attractive interaction the gas-liquid transition of a given substance becomes metastable with respect to the fluid-solid transition. ${ }^{1-10}$ This was first realized and treated theoretically in connection with phase transitions in mixed suspensions of colloidal particles and nonadsorbing polymer molecules by Gast, Hall, and Russel. ${ }^{11}$ In such colloidpolymer mixtures, the range and the depth of the attractive interaction can be adjusted by the size and concentration of the added polymer molecules. ${ }^{12-14}$ Experiments on model colloid-polymer mixtures have provided conclusive evidence that the topology of the phase diagram is indeed determined by the ratio of the radius of gyration of the polymer molecules to the radius of the colloidal particles. ${ }^{15-19}$ For sufficiently large values of this ratio $(\geqslant 0.25)$ Ilett et al. ${ }^{18}$ observe a three-phase coexistence region of colloidal gas, colloidal liquid, and colloidal crystal phases bordered by three two-phase regions, colloidal-gas + colloidal liquid, colloidal-liquid + colloidal-crystal, colloidal-gas + colloidal crystal, whereas for small values of this ratio $(\leqslant 0.25)$ the addition of the polymer only expands the colloidal fluidcolloidal crystal region of the phase diagram of the pure colloidal system, as predicted by Lekkerkerker et $a l^{20}$

Following the work on colloid-polymer mixtures, it was subsequently recognized that sufficiently short-ranged attractions could also lead to gas-liquid metastability in molecular systems. An example of such a molecular system that has emerged in recent years is the $\mathrm{C}_{60}$ molecule. Hagen et al. ${ }^{21}$ mapped out the phase diagram of $\mathrm{C}_{60}$ using computer simulations in which the $\mathrm{C}_{60}$ molecules are represented by spheres interacting via the Girifalco potential. ${ }^{22}$ They concluded that $\mathrm{C}_{60}$ has no stable liquid phase. Cheng et al., ${ }^{23}$ also starting from the Girifalco potential, used an integralequation approach combined with molecular dynamics simulations to establish the phase diagram of $\mathrm{C}_{60}$. They predicted that the liquid phase is stable in a narrow range of temperatures and densities. Subsequent theoretical work has not defi-

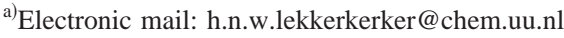

nitely settled this point. ${ }^{8,9,24,25}$ Also, this issue has not yet been experimentally resolved. ${ }^{26}$ Altogether, these results suggest that $\mathrm{C}_{60}$ is a borderline case which may or may not have a liquid phase.

The example of $\mathrm{C}_{60}$ inspired several systematic attempts, both theoretical and numerical to establish the relation between the range of the attractive part of the intermolecular potential and the stability of the liquid phase. ${ }^{1-9}$ These calculations confirmed that below a critical range of the attraction the liquid state becomes metastable.

For solutions of globular proteins, it has been known for more than 20 years that a liquid-liquid phase separation occurs below the fluid-solid transition, i.e., this liquid-liquid phase separation is metastable. ${ }^{27-36}$ In the last few years, it was realized that a possible explanation for the occurrence of this metastable liquid-liquid phase separation is again the fact that the range of attraction is small compared to the size of the protein molecule. ${ }^{37-43}$

The metastable gas-liquid or liquid-liquid phase separation appears to have a considerable effect on the crystallization behavior. This was first observed empirically in the case of colloid-polymer mixtures. ${ }^{44-46}$ The connection with the presence of a metastable colloidal gas-colloidal liquid transition was made by Poon et al. ${ }^{47}$ and treated theoretically by Evans, Poon, and Cates. ${ }^{48}$ In the last two years, experiments, ${ }^{35,36}$ numerical calculations, ${ }^{49}$ and theoretical considerations $^{50}$ have been reported which show that the presence of a metastable liquid-liquid phase separation drastically changes the crystallization pathway in solutions of globular proteins. Furthermore, a relation is emerging between the second virial coefficient as a predictor for protein crystallization $^{51,52}$ and the solubility of proteins in aqueous solution. ${ }^{43,53}$ These developments led us to investigate the relation between the appearance of the gas-liquid phase separation as marked by the critical temperature and the second virial coefficient. Using simulation results for a variety of potentials with a varying range of attraction, we established that whereas the critical temperature drops considerably upon narrowing the range of attraction, the second virial coefficient at the critical temperature remains practically constant. This allows us to predict the critical temperature 
TABLE I. Computer simulation results for $T_{c}, \rho_{c}$, and calculated values for $B_{2}$ at $T_{c}$ for the square well model. The simulation results were taken from Ref. 56.

\begin{tabular}{llll}
\hline \hline \multicolumn{1}{c}{$\lambda$} & $k_{B} T_{c} / \epsilon$ & $\rho_{c} \sigma^{3}$ & $B_{2}\left(T_{c}\right) / v_{0}$ \\
\hline 2.0 & 2.764 & 0.225 & -8.21 \\
1.75 & 1.811 & 0.284 & -8.85 \\
1.5 & 1.219 & 0.299 & -8.85 \\
1.375 & 0.974 & 0.355 & -7.46 \\
1.25 & 0.764 & 0.37 & -6.30 \\
\hline \hline
\end{tabular}

for any "simple" isotropic potential, i.e., consisting of a steep repulsion at short distances and an attraction for larger distances. Furthermore, we discuss the connection between the second virial coefficient as a predictor for the critical temperature and the second virial coefficient as predictor in crystal growth as was proposed by George and Wilson. ${ }^{51,52}$

\section{LOCATION OF THE CRITICAL POINT FOR DIFFERENT POTENTIALS WITH A VARIABLE RANGE OF ATTRACTION}

Using different simulation techniques, notably the Gibbs ensemble Monte Carlo method, ${ }^{54}$ in recent years the gasliquid part of the phase diagram of a number of potentials with different range of attraction has been determined. The model systems under consideration here are the square well fluid, hard-core Yukawa fluid and the Lennard-Jones $2 n$ $-n$ fluid. These potentials are all isotropic. In the Secs. II A-II C, we discuss the above-mentioned model potentials (for various ranges) and give their critical temperature and the second virial coefficient at the critical temperature $B_{2}\left(T_{c}\right)$.

At a given temperature, the second virial coefficient

$$
B_{2}(T)=2 \pi \int_{0}^{\infty}\left(1-\exp \left(-\frac{V(r)}{k_{B} T}\right)\right) r^{2} d r
$$

where $V(r)$ is the pair potential, $k_{B}$ the Boltzmann constant, and $T$ the absolute temperature, is a measure for the relative importance of attractive interactions. A positive $B_{2}(T)$ indicates repulsions to be dominant, whereas a negative $B_{2}(T)$ indicates that attractions are important. In the following, $B_{2}$ is expressed in units $v_{0}=(\pi / 6) \sigma^{3}$, the volume of the particles. The diameter $\sigma$ is in all cases taken to be the distance at which the potential crosses zero.

\section{A. Square well}

The square well potential is a well-studied model system in liquid state physics. ${ }^{55}$ Although the model is simple, it contains both a repulsive and an attractive part in the potential which is necessary (and sufficient) to exhibit a gasliquid phase transition. The square well potential is given by

$$
V(r)=\left\{\begin{array}{l}
\infty \quad \text { if } r<\sigma, \\
-\epsilon \quad \text { if } \sigma \leqslant r<\lambda \sigma, \\
0 \quad \text { if } r \geqslant \lambda \sigma .
\end{array}\right.
$$

Here, $\lambda$ is a measure for the range of the potential and $\epsilon$ is the depth of the potential. Larger values of $\lambda$ correspond to wider potential wells. In Table I the critical constants as
TABLE II. Simulation results for $T_{c}, \rho_{c}$ and calculated values of $B_{2}$ at $T_{c}$ for the hard-core Yukawa model. The simulation results of $\kappa \sigma=1.8,3.0$, and 4.0 were taken from Ref. 57 and those for $\kappa \sigma=3.9$ and 7.0 from Ref. 2 .

\begin{tabular}{llll}
\hline \hline$\kappa \sigma$ & $k_{B} T_{c} / \epsilon$ & $\rho_{c} \sigma^{3}$ & $B_{2}\left(T_{c}\right) / v_{0}$ \\
\hline 1.8 & 1.17 & 0.313 & -6.23 \\
3.0 & 0.715 & 0.375 & -6.12 \\
4.0 & 0.576 & 0.377 & -5.90 \\
3.9 & 0.55 & 0.370 & -6.94 \\
7.0 & 0.412 & 0.500 & -5.45 \\
\hline \hline
\end{tabular}

determined from simulations ${ }^{56}$ and the corresponding values of $B_{2}\left(T_{c}\right)$ as calculated by us are given for a number of square well potentials with different ranges.

\section{B. Hard-core Yukawa}

The hard-core Yukawa potential is characterized by a hard-sphere core and an exponential attraction

$$
V(r)=\left\{\begin{array}{l}
\infty \quad \text { if } r<\sigma, \\
-\epsilon \frac{e^{\kappa \sigma(1-r / \sigma)}}{r / \sigma} \quad \text { if } r \geqslant \sigma .
\end{array}\right.
$$

The range of attraction is tuned by the parameter $\kappa$, which has the dimension of reciprocal length. For larger $\kappa \sigma$ the potential becomes more narrow. In Table II the critical constants for different $\kappa \sigma$, following from simulations, ${ }^{2,57}$ and the corresponding $B_{2}\left(T_{c}\right)$ as calculated by us are given.

\section{Lennard-Jones $2 n-n$}

The Lennard-Jones potentials

$$
V(r)=4 \epsilon\left[\left(\frac{\sigma}{r}\right)^{2 n}-\left(\frac{\sigma}{r}\right)^{n}\right],
$$

constitute another widely studied family of interaction potentials. Here, $\sigma$ is the position at which the potential crosses zero and $\epsilon$ the depth of the potential. With increasing $n$, this potential becomes more steeply repulsive and more narrowly attractive. In Table III the critical points of the gas-liquid transition as determined by computer simulation ${ }^{10}$ and the corresponding values of $B_{2}\left(T_{c}\right)$ are listed for a number of these potentials. From the data presented above it appears that $B_{2}\left(T_{c}\right)$ lies in a fairly narrow range around $-6.3 v_{0}$. This leads us to think that whenever $B_{2}$ is about $-6 v_{0}$, the fluid must be close to its critical temperature.

\section{Illustrative examples}

Let us now apply the criterion $B_{2}\left(T_{c}\right)=-6 v_{0}$ to two well-studied models which have a potential formed by a steep repulsion at short distances and an attraction for longer distances but with a different functional form when compared to the potentials discussed above.

The first case concerns the Girifalco potential for $\mathrm{C}_{60},{ }^{22}$ which was already mentioned in Sec. I. Here, the pair potential between two $\mathrm{C}_{60}$ molecules is constructed by summing all the carbon-carbon interactions between the two molecules assuming that these interact through a Lennard-Jones potential. This results in the following pair potential: 
TABLE III. The critical point and $B_{2}$ at the critical point for the LennardJones $2 n-n$ model for various values of $n$ (Ref. 11).

\begin{tabular}{rccc}
\hline \hline$n$ & $k_{B} T_{c} / \epsilon$ & $\rho_{c} \sigma^{3}$ & $B_{2}\left(T_{c}\right) / v_{0}$ \\
\hline 6 & 1.316 & 0.304 & -6.19 \\
7 & 0.997 & 0.317 & -6.43 \\
8 & 0.831 & 0.326 & -6.45 \\
9 & 0.730 & 0.354 & -6.36 \\
12 & 0.560 & 0.378 & -6.36 \\
18 & 0.425 & 0.420 & -6.41 \\
\hline
\end{tabular}

$$
\begin{aligned}
V(r)= & -\alpha\left[\frac{1}{s(s-1)^{3}}+\frac{1}{s(s+1)^{3}}-\frac{2}{s^{4}}\right] \\
& +\beta\left[\frac{1}{s(s-1)^{9}}+\frac{1}{s(s+1)^{9}}-\frac{2}{s^{10}}\right] .
\end{aligned}
$$

Here, $s=r / 2 a$, with $a=0.355 \mathrm{~nm}$ and $\alpha$ and $\beta$ are interaction constants which are $7.494 \times 10^{-21} \mathrm{~J}$ and $1.360 \times 10^{-23} \mathrm{~J}$, respectively. The potential has a minimum $\epsilon=-44.43$ $\times 10^{-21} \mathrm{~J}$ at $r / 2 a=1.415$, is zero at $r / 2 a=1.35$, and becomes infinite for $r / 2 a=1$. The critical temperature obtained from simulations is $k_{B} T_{c} / \epsilon=0.558 .{ }^{21}$ The $B_{2}=-6 v_{0}$ criterion predicts $k_{B} T_{c} / \epsilon=0.596$, differing $7 \%$ from the simulation value.

Our second example concerns the potential used by Ten Wolde and Frenkel ${ }^{49}$ in their study of the nucleation pathways in protein solutions

$$
V(r)=\frac{4 \epsilon}{\alpha^{2}}\left[\frac{1}{\left[\left(\frac{r}{2 a}\right)^{2}-1\right]^{6}}-\alpha \frac{1}{\left[\left(\frac{r}{2 a}\right)^{2}-1\right]^{3}}\right] .
$$

This potential can be considered as a generalized LennardJones model and has a minimum $\epsilon=-1$ at $r / 2 a$ $=\sqrt{(2 / \alpha)^{1 / 3}+1}$, is zero at $r / 2 a=\sqrt{(1 / \alpha)^{1 / 3}+1}$, and diverges for $r / 2 a=1$. The parameter $\alpha$ tunes the attractive range and by increasing $\alpha$ the potential becomes more narrow. For $\alpha=50$, the critical temperature was determined from simulations to be $k_{B} T_{c} / \epsilon=0.418$. $^{49}$ The prediction from the $B_{2}$ $=-6 v_{0}$ criterion gives $k_{B} T_{c} / \epsilon=0.412$, differing $1 \%$.

The above examples give us confidence that for systems interacting through simple continuous potentials, the $B_{2}$ $=-6 v_{0}$ criterion is a good predictor for the critical temperature.

The constancy of $B_{2}\left(T_{c}\right)$ should perhaps not come as a surprise when considering the two classical limiting cases for the interaction range which are described by the van der Waals model ${ }^{58}$ (long-ranged attractions) and the Baxter model $^{59}$ (very short ranged attractions). In the van der Waals model, the equation of state consists of a hard-sphere repulsive part which can be described by the scaled particle equation of state ${ }^{60}$ plus a mean field contribution due to longranged attractive interactions. This results in the following equation of state:

$$
P=k_{B} T \frac{\rho\left(1+\phi+\phi^{2}\right)}{(1-\phi)^{3}}-a \rho^{2} .
$$

TABLE IV. The critical point calculated using a virial expansion up to $B_{4}$. The virial coefficients are given in the appropriate powers of the eigenvolume $v_{0}$ of the particles.

\begin{tabular}{rccccc}
\hline \hline$n$ & $k_{B} T_{c} / \epsilon$ & $\rho_{c} \sigma^{3}$ & $B_{2}\left(T_{c}\right) / v_{0}$ & $B_{3}\left(T_{c}\right) / v_{0}^{2}$ & $B_{4}\left(T_{c}\right) / v_{0}^{3}$ \\
\hline 6 & 1.300 & 0.268 & -6.34 & 9.41 & 20.3 \\
7 & 0.980 & 0.252 & -6.66 & 9.93 & 26.7 \\
8 & 0.810 & 0.243 & -6.83 & 9.77 & 31.5 \\
9 & 0.705 & 0.239 & -6.91 & 9.77 & 34.4 \\
12 & 0.548 & 0.241 & -6.79 & 8.93 & 35.1 \\
18 & 0.426 & 0.259 & -6.31 & 7.64 & 29.2 \\
\hline \hline
\end{tabular}

Here, $\rho$ is the number density and $\phi=\rho v_{0}$ the volume fraction in the system. If we calculate the critical point from this equation of state, we find $k_{B} T_{c} /\left(a / \sigma^{3}\right)=0.18$ and $B_{2}\left(T_{c}\right)$ $=-6.66 v_{0}$.

The short-range attraction limit given by the Baxter potential

$$
\frac{V(r)}{k_{B} T}=\lim _{\lambda \rightarrow 1}\left\{\begin{array}{l}
\infty \quad \text { if } r<\sigma, \\
\ln \left(\frac{12 \tau(\lambda-1) \sigma}{\lambda \sigma}\right) \quad \text { if } \sigma \leqslant r<\lambda \sigma, \\
0 \quad \text { if } r \geqslant \lambda \sigma .
\end{array}\right.
$$

is essentially a square well model in the simultaneous limit $\lambda \rightarrow 1$ and $\epsilon \rightarrow-\infty$ while keeping $B_{2}$ finite.

$$
B_{2}=\frac{\pi}{6} \sigma^{3}\left[4-\frac{1}{\tau}\right] .
$$

The pair correlation function for this model potential can be solved analytically within the Percus-Yevick approximation. However, since the Percus-Yevick equation is not exact, the resulting equation of state depends on whether it is calculated from the compressibility equation or via the internal energy. The critical values of the Baxter $\tau$ parameter and $B_{2}\left(\tau_{c}\right)$ are given by $\tau_{c}=0.0976$ and $B_{2}\left(\tau_{c}\right)=-6.20 v_{0}$ (compressibility equation) and $\tau_{c}=0.1185$ and $B_{2}\left(\tau_{c}\right)$ $=-4.33 v_{0}$ (energy equation). ${ }^{61}$ So indeed, the $B_{2}$ values at the critical point for both the van der Waals equation and the Baxter potential lie in the same range as the results obtained for the model potentials discussed in the Secs. II A-II D.

A second attempt to "understand" the constancy of $B_{2}\left(T_{c}\right)$ was made by calculating the critical point using a virial expansion up to fourth order for the Lennard-Jones $2 n-n$ potentials. Such calculations were already carried out more than 30 years ago by Barker and Monaghan ${ }^{62}$ for the Lennard-Jones 12-6 potential. The results of those calculations, $k_{B} T_{c} / \epsilon=1.300$ and $\rho_{c} \sigma^{3}=0.294$, compare well with the simulation data $k_{B} T_{c} / \epsilon=1.316$ and $\rho_{c} \sigma^{3}=0.301 .{ }^{63} \mathrm{In}$ spired by Barker's work we have calculated the critical point for several Lennard-Jones $2 n-n$ potentials. From the results listed in Table IV, using a virial expansion of the pressure up to $B_{4}$, we note that this simple treatment reproduces quite well the critical temperatures and hence the constancy of the second virial coefficient. However, the critical density is not well reproduced and even shows the wrong trend. 

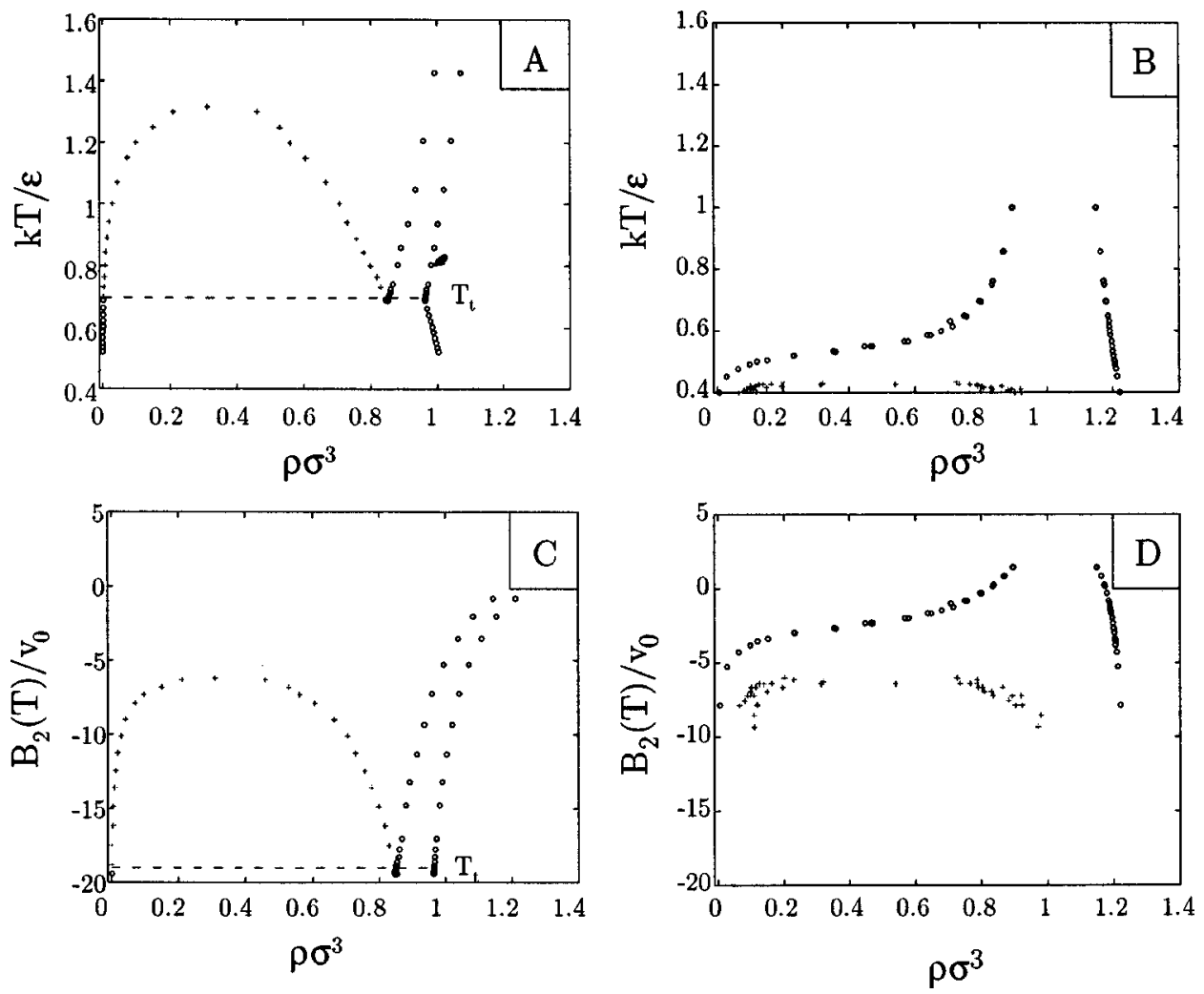

FIG. 1. Phase diagrams for the Lennard-Jones $12-6$ and $36-18$ potentials in the $\rho \sigma^{3}-k_{B} T / \epsilon(\mathrm{A})$ and (B) and the $\rho \sigma^{3}-B_{2}(\mathrm{C})$ and (D) representation.

\section{THE SECOND VIRIAL COEFFICIENT AS PREDICTOR IN PROTEIN CRYSTAL GROWTH}

George and Wilson ${ }^{51,52}$ observed that for protein solutions which are suitable for crystal growth, the osmotic second virial coefficient $B_{2}$ falls in a narrow range, the so-called crystallization slot. It is therefore interesting to plot phase diagrams in the $B_{2}$-density representation and compare them to the usual temperature density representation. In Fig. 1 this is done for the Lennard-Jones 12-6 (long-ranged attraction) and 36-18 (short-ranged attraction) potentials.

In the temperature-density plane, the appearance of the (metastable) gas-liquid transition is quite sensitive to the interaction range, whereas in the $B_{2}$-density plane the (metastable) gas-liquid boundary is bounded by $B_{2} \simeq-6 v_{0}$. While simple liquids typically have a phase diagram given of the type in Fig. 1(A), it has been argued that the phase dia-

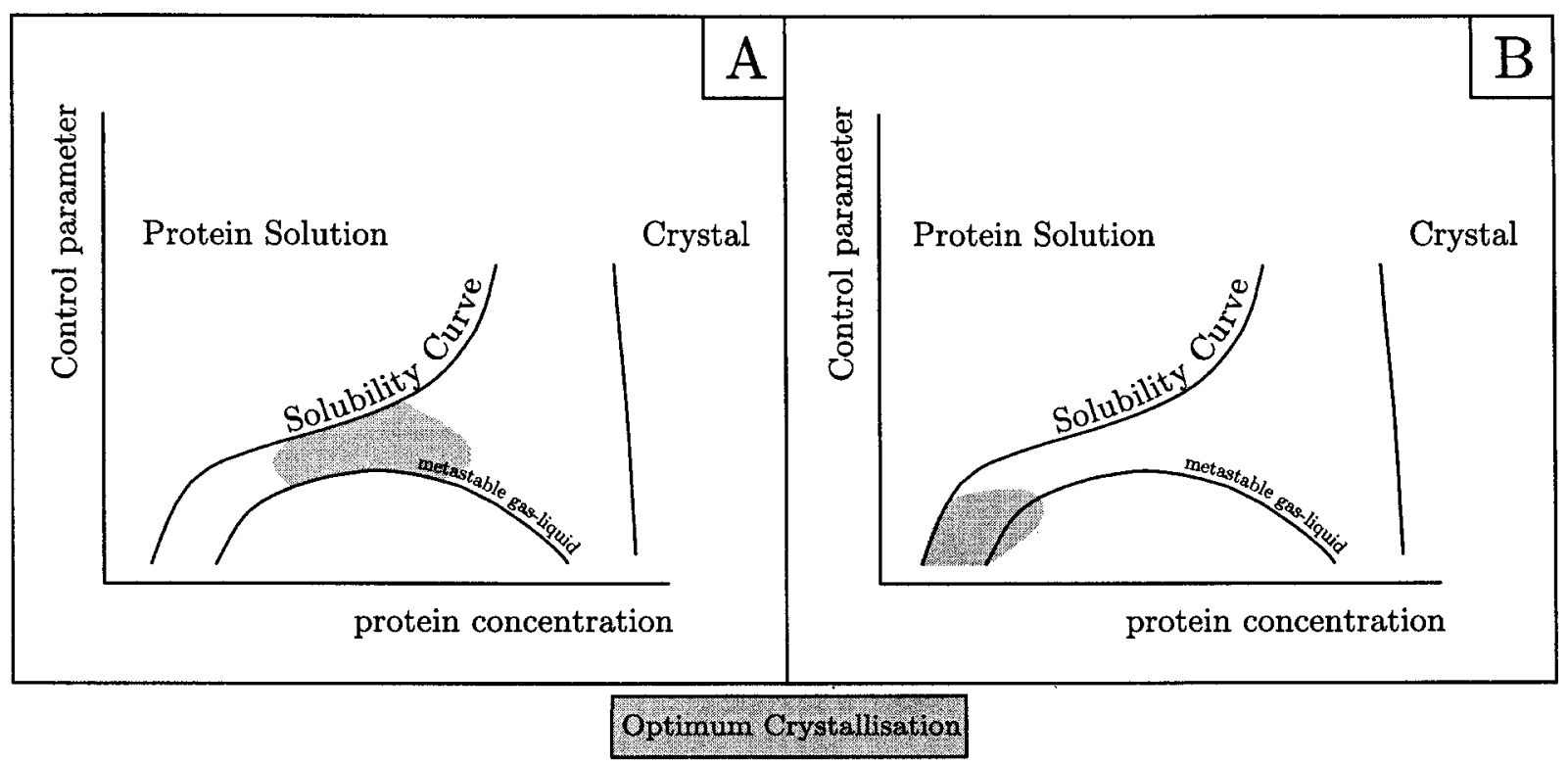

FIG. 2. Schematic phase diagrams indicating the regions of optimum crystallization. (A) Optimum crystallization around the critical point. (B) Optimum crystallization below the critical point. 
TABLE V. The second virial coefficient for a number of proteins, data taken from Ref. 43.

\begin{tabular}{lrcc}
\hline \multicolumn{1}{c}{ Protein } & Mass $(\mathrm{kD})$ & $B_{2}\left(10^{-4} \mathrm{~mol} \mathrm{~cm}^{3} \mathrm{~g}^{-2}\right)$ & $B_{2} / v_{0}$ \\
\hline BSA & 65 & -2.1 & -18.6 \\
Canavalin & 141 & -0.8 & -15.3 \\
Concanavalin A & 102 & -2.5 & -34.7 \\
Concanavalin A & 102 & -1.9 & -26.4 \\
$\alpha$-chymotrypsin & 25 & -8.4 & -28.6 \\
$\alpha$-lactalbumin & 14 & -7.3 & -13.9 \\
$\beta$-lactoglobulin A & 36 & -2.4 & -11.8 \\
$\beta$-lactoglobulin A & 36 & -6.2 & -30.4 \\
$\beta$-lactoglobulin B & 36 & -2.8 & -13.7 \\
$\beta$-lactoglobulin B & 36 & -6.2 & -30.4 \\
Lysozyme & 14 & -2.8 & -5.3 \\
Ovostatin & 720 & -7.1 & -695 \\
Ovalbumin & 43 & -6.1 & -35.7 \\
Pepsin & 36 & -7.8 & -38.2 \\
Pepsin & 36 & -2.8 & -13.7 \\
Pepsin & 36 & -0.8 & -3.9 \\
Ribonuclease A & 14 & -4.1 & -7.8 \\
STMV & 1500 & -1.8 & -367 \\
Thaumatin & 22 & -3.0 & -9.0 \\
\hline \hline
\end{tabular}

gram given in Fig. 1(B) applies to protein solutions. ${ }^{37,49}$ Furthermore, it has been proposed that in the case of a metastable gas-liquid region in the phase diagram, crystal nucleation will occur via a two-step process. ${ }^{49,53}$ The first step is the formation of small regions (droplets) with a high protein concentration. These regions can be either critical concentration fluctuations ${ }^{49}$ or droplets associated with the metastable liquid-liquid phase separation. ${ }^{53}$ The second step in the nucleation process is the formation of small crystals in the regions of high protein concentration. In Fig. 2 we schematically indicate the corresponding conditions for optimum protein crystallization.

In Fig. 2(A), we consider the case in which the enhancement of protein crystal nucleation is driven by critical concentration fluctuations, whereas in Fig. 1(B), the crystal nucleation process starts with the formation of protein-rich droplets below the metastable critical point

If we now make the bold assumption that the (metastable) liquid-liquid demixing critical point in solutions of globular proteins is also located at conditions where the osmotic second virial coefficient is $-6 v_{0}$, we can connect the optimum crystallization conditions collected by George and Wilson $^{51,52}$ to a region in the phase diagram. The optimum crystallization conditions indicated in Fig. 2(A) then correspond to conditions $B_{2} / v_{0} \simeq-6$ while the conditions in Fig. 2(B) are related to $B_{2} / v_{0}<-6$. In order to make the link between our phase diagrams and $B_{2}$ data of George and Wilson, ${ }^{51,52}$ we have converted their data, which are expressed in the usual units $\mathrm{mol} \mathrm{cm} \mathrm{c}^{3} \mathrm{~g}^{-2}$ units, to $v_{0}$ units used in our approach. This can be done by the simple relation, ${ }^{43}$

$$
\frac{B_{2}}{v_{0}}=B_{2}^{\prime} d M,
$$

where $B_{2}^{\prime}$ is the second virial coefficient in units $\mathrm{mol} \mathrm{cm} \mathrm{g}^{3}, d$ is the average mass density of the proteins and $M$ their molar mass. Using the mass density $d$ $=1.36 \mathrm{~g} / \mathrm{cm}^{3}$ and the molecular weights given by Haas and
Drenth, ${ }^{43}$ we obtain the results presented in Table V. From Table $\mathrm{V}$ we note that apart from two exceptionally large negative values of $B_{2} / v_{0}$ for STMV and ovastatin, the second virial coefficients vary between $-4 v_{0}$ and $-40 v_{0}$. The values $B_{2} / v_{0}$, which are on average smaller than -6 indicate that the optimum crystallization conditions vary from around the critical point to below the critical point.

\section{ACKNOWLEDGMENTS}

The authors wish to thank Jan Dhont for many stimulating discussions and help on the calculation of the virial coefficients. We are grateful to Daan Frenkel, Paul van der Schoot and Ben Widom for helpful discussions and sharing their insights on critical phenomena. Finally we thank Jan Drenth, Cor Haas, and Roberto Piazza for sending us preprints of their work and for illuminating discussions on protein crystallization.

${ }^{1}$ M. H. J. Hagen and D. Frenkel, J. Chem. Phys. 101, 4093 (1994)

${ }^{2}$ L. Mederos and G. Navacués, J. Chem. Phys. 101, 9841 (1994).

${ }^{3}$ K. Shukla and R. Rajagopalan, J. Chem. Phys. 101, 11077 (1994).

${ }^{4}$ C. F. Tejero, A. Daanoun, H. N. W. Lekkerkerker, and M. Baus, Phys. Rev. Lett. 73, 752 (1994).

${ }^{5}$ C. F. Tejero, A. Daanoun, H. N. W. Lekkerkerker, and M. Baus, Phys. Rev. E 51, 558 (1995).

${ }^{6}$ J. P. K. Doyle and D. J. Wales, J. Phys. B 29, 4859 (1996).

${ }^{7}$ M. Hasegawa and K. Ohno, Phys. Rev. E 54, 3928 (1996).

${ }^{8}$ M. Hasegawa and K. Ohno, J. Phys.: Condens. Matter 9, 3361 (1997).

${ }^{9}$ M. Hasegawa, J. Chem. Phys. 108, 208 (1998).

${ }^{10}$ G. A. Vliegenthart, J. F. M. Lodge, and H. N. W. Lekkerkerker, Physica A 263, 378 (1999).

${ }^{11}$ A. P. Gast, C. K. Hall, and W. B. Russel, J. Colloid Interface Sci. 96, 251 (1983).

${ }^{12}$ S. Asakura and F. Oosawa, J. Chem. Phys. 22, 1255 (1954).

${ }^{13}$ S. Asakura and F. Oosawa, J. Polym. Sci. 33, 183 (1958).

${ }^{14}$ A. Vrij, Pure Appl. Chem. 48, 471 (1976).

${ }^{15}$ B. Vincent, J. Edwards, S. Emmet, and R. Croot, Colloids Surface 31, 267 (1988).

${ }^{16}$ F. Leal Calderon, J. Bibette, and J. Bias, Europhys. Lett. 23, 653 (1993).

${ }^{17}$ P. N. Pusey, W. C. K. Poon, S. M. Ilett, and P. Bartlett, J. Phys.: Condens. Matter 6, A29 (1994).

${ }^{18}$ S. M. Ilett, A. Orrock, W. C. K. Poon, and P. N. Pusey, Phys. Rev. E 51, 1344 (1995)

${ }^{19}$ A. Moussaï, W. C. K. Poon, P. N. Pusey, and M. F. Soliva, Phys. Rev. Lett. 82, 225 (1999)

${ }^{20}$ H. N. W. Lekkerkerker, W. C. K. Poon, P. N. Pusey, A. Stroobants, and P. B. Warren, Europhys. Lett. 20, 559 (1992).

${ }^{21}$ M. H. J. Hagen, E. J. Meijer, G. C. A. M. Mooij, D. Frenkel, and H. N. W. Lekkerkerker, Nature (London) 365, 425 (1993).

${ }^{22}$ L. A. Girifalco, J. Phys. Chem. 96, 858 (1992).

${ }^{23}$ A. Cheng, M. L. Klein, and C. Caccamo, Phys. Rev. Lett. 71, 1200 (1993).

${ }^{24}$ L. Mederos and G. Navacués, Phys. Rev. B 50, 1301 (1994).

${ }^{25}$ C. Caccamo, Phys. Rev. B 51, 3387 (1995).

${ }^{26}$ P. C. Ecklund, M. S. Dresselhaus, and G. Dresselhaus, Science of Fullerenes and Carbon Nanotubes (Academic, San Diego, 1996).

${ }^{27}$ C. Ishimoto and T. Tanaka, Phys. Rev. Lett. 39, 474 (1977).

${ }^{28}$ G. D. J. Phillies, Phys. Rev. Lett. 55, 1341 (1985).

${ }^{29}$ V. G. Taratuta, A. Holschbach, G. M. Thurston, D. Blankschtein, and G. B. Benedek, J. Phys. Chem. 94, 2140 (1990).

${ }^{30}$ M. L. Broide, T. M. Tominic, and M. D. Saxowsky, Phys. Rev. E 53, 6325 (1996).

${ }^{31}$ J. A. Thomson, P. Schurtenberger, G. M. Thurston, and G. B. Benedek, Phys. Rev. A 84, 7079 (1987).

${ }^{32}$ P. Schurtenberger, R. A. Chamberlin, G. M. Thurston, J. A. Thomson, and G. B. Benedek, Phys. Rev. Lett. 63, 2064 (1989).

${ }^{33}$ M. L. Broide, C. R. Berland, J. Pande, O. O. Ogun, and G. B. Benedek, Proc. Natl. Acad. Sci. USA 88, 5660 (1991).

${ }^{34}$ C. R. Berland, G. M. Thurston, M. Kondo, M. Broide, J. Pande, O. O. 
Ogun, and G. B. Benedek, Proc. Natl. Acad. Sci. USA 89, 1214 (1992).

${ }^{35}$ M. Muschol and F. Rosenberger, J. Chem. Phys. 107, 1953 (1997).

${ }^{36}$ S. Tanaka, M. Yamamoto, K. Ito, R. Hayakawa, and M. Ataka, Phys. Rev. E 56, R67 (1997).

${ }^{37}$ D. Rosenbaum, P. C. Zamora, and C. F. Zukoski, Phys. Rev. Lett. 76, 150 (1996).

${ }^{38}$ A. Lomakin, N. Asherie, and G. B. Benedek, J. Chem. Phys. 104, 1646 (1996).

${ }^{39}$ N. Asherie, A. Lomakin, and G. B. Benedek, Phys. Rev. Lett. 77, 4832 (1996).

${ }^{40}$ M. Malfois, F. Bonneté, L. Belloni, and A. Tardieu, J. Chem. Phys. 105, 3290 (1996).

${ }^{41}$ W. C. K. Poon, Phys. Rev. E 55, 3762 (1997).

${ }^{42}$ R. Piazza, V. Peyre, and V. Degiorgio, Phys. Rev. E 58, R2733 (1998).

${ }^{43}$ C. Haas and J. Drenth, J. Phys. Chem. B 102, 4226 (1998).

${ }^{44}$ C. Smits, J. S. van Duijneveldt, J. K. G. Dhont, H. N. W. Lekkerkerker, and W. Briels, Phase Transit. 21, 157 (1990).

${ }^{45}$ C. Smits, B. van der Most, J. K. G. Dhont, and H. N. W. Lekkerkerker, Adv. Colloid Interface Sci. 42, 33 (1992).

${ }^{46}$ P. Pusey, Physica A 201, 322 (1993).

${ }^{47}$ W. C. K. Poon, A. D. Pirie, and P. N. Pusey, Faraday Discuss. 101, 65 (1995).
${ }^{48}$ R. M. L. Evans, W. C. K. Poon, and M. E. Cates, Europhys. Lett. 38, 595 (1997).

${ }^{49}$ P. R. Ten Wolde and D. Frenkel, Science 277, 1975 (1997).

${ }^{50}$ V. Talanquer and D. Oxtoby, J. Chem. Phys. 109, 223 (1998).

${ }^{51}$ A. George and W. W. Wilson, Acta Crystallogr., Sect. D: Biol. Crystallogr. 50, 361 (1994).

${ }^{52}$ A. George, Y. Ciang, B. Guo, A. Arabshahi, Z. Cai, and W. W. Wilson, Methods Enzymol. 276, 100 (1997).

${ }^{53}$ C. Haas and J. Drenth, J. Cryst. Growth 196, 388 (1999).

${ }^{54}$ A. Z. Panagiotopoulos, Mol. Phys. , 61, 813 (1987).

${ }^{55}$ E. A. Mason and T. H. Spurling, The Virial Equation of State (Pergamon, Oxford, 1969).

${ }^{56}$ L. Vega, E. de Miguel, L. F. Rull, G. Jackson, and I. A. McLure, J. Chem. Phys. 96, 2296 (1992).

${ }^{57}$ E. Lomba and N. G. Almarza, J. Chem. Phys. 100, 8367 (1994).

${ }^{58}$ H. C. Longuet-Higgins and B. Widom, Mol. Phys. 8, 549 (1964).

${ }^{59}$ R. J. Baxter, J. Chem. Phys. 49, 2770 (1968).

${ }^{60}$ H. Reiss, H. L. Frisch, and J. C. Lebowitz, J. Chem. Phys. 31, 369 (1959).

${ }^{61}$ R. O. Watts, D. Henderson, and R. J. Baxter, Adv. Chem. Phys. 21, 412 (1971).

${ }^{62}$ J. A. Barker and J. J. Monaghan, J. Chem. Phys. 36, 2564 (1962).

${ }^{63}$ B. Smit and C. P. Williams, J. Phys.: Condens. Matter 2, 4281 (1990). 